\title{
Erratum
}

\section{On the representation of integers by binary cubic forms of positive discriminant}

\author{
J.H. Evertse
}

Invent. math. (1983) $73,117-138$

On page 120 of the above article I derived Theorem 1 from Theorem 3 by showing the following:

Proposition. Let $F(x, y) \in \mathbb{Z}[x, y]$ be a binary cubic form of positive discriminant which is reduced and irreducible over $\mathbb{Q}$. Then the equation

$$
F(x, y)=1 \text { in } x, y \in \mathbb{Z}
$$

has at most three solutions with $|x y| \leqq 1$.

Our proof of this proposition was not correct and in fact the proposition is not true. For if $F$ is equal to the reduced form $F_{1}(x, y)=x^{3}+x^{2} y-2 x y^{2}-y^{3}$ of discriminant 49 then (1) has the solutions $(x, y)=(1,0),(0,-1),(-1,1),(-1$, -1). But Baulin showed (cf. p. 117 of the above article) that (1) has exactly nine solutions if $F=F_{1}$ and this is better than the result of Theorem 1 .

We shall now give a correct proof of the proposition, however under the assumptions that $F$ has positive discriminant and is not equivalent to $F_{1}$. This suffices to prove Theorem 1.

Similar to the arguments on p. 120 of the above article we may assume that $F(1,0)=1$ and that (1) has two solutions with $|x| \leqq 1$ and $y=1$. Then

$$
F(x, y)=(x-p y)(x-q y)(x-r y)+y^{3} \quad \text { with } p, q \in\{-1,0,1\}, p>q, r \in \mathbb{Z} .
$$

If (1) has three solutions with $y=1,|x| \leqq 1$ then $|r| \leqq 1, r \neq p, r \neq q$. But then $F$ is a form of discriminant -23 which case was excluded. If (1) has a solution with $|x| \leqq 1, y=-1$ then

$$
(x+p)(x+q)(x+r)=2 .
$$

If $x=-1$ then $p=0, q=-1, r=2$ and $F(x, y)=F_{1}(x,-y)$. If $x=0$ then $p=1, q$ $=-1, r=-2$ and $F$ has discriminant -87 . Finally, if $x=1$ then $p=1, q=0, r$ $=0$ and $F$ has discriminant -23 . Hence if $F$ is of type (2) then the only solutions of (1) with $|x y| \leqq 1$ are $(1,0),(p, 1),(q, 1)$. This proves the proposition, under the restrictions that $F$ has positive discriminant and is not equivalent to $F_{1}$. 